\title{
Atypical fractures do not have a thicker cortex
}

\author{
V A Koeppen, Jörg Schilcher and Per Aspenberg
}

\section{Linköping University Post Print}

N.B.: When citing this work, cite the original article.

The original publication is available at www.springerlink.com:

V A Koeppen, Jörg Schilcher and Per Aspenberg, Atypical fractures do not have a thicker cortex, 2012, Osteoporosis International, (23), 12, 2893-2896.

http://dx.doi.org/10.1007/s00198-012-2173-9

Copyright: Springer Verlag (Germany)

http://www.springerlink.com/?MUD=MP

Postprint available at: Linköping University Electronic Press

http://urn.kb.se/resolve?urn=urn:nbn:se:liu:diva-86631 


\title{
Atypical fractures do not have a thicker cortex
}

\author{
Veronika A. Koeppen, Jörg Schilcher, Per Aspenberg
}

Orthopedics, Department of clinical and experimental medicine, Faculty of health science, Linköping University, Sweden.

Corresponding author: Per Aspenberg

Phone: $+46(0) 10-1034166$

E-mail: per.aspenberg@liu.se

Address:

Orthopedics

Department of Clinical and Experimental Medicine

Linköping University

S-581 85 Linköping, Sweden 


\section{Disclosure}

The study was funded by the Swedish Research Council (VR 2009-6725), Linköping University, Östergötland County Council, and the King Gustaf V and Queen Victoria Free Mason Foundation.

PA has a patent on a process for coating metal implants with bisphosphonates, and shares in a company (Addbio $\mathrm{AB}$ ) trying to commercialize the principle. PA also has received consulting reimbursement and grants from Eli Lilly \& Co.

VK and JS have no conflict of interest to declare. 


\title{
Mini-abstract
}

An association between atypical fractures and general cortical thickness of the femoral shaft is often suggested in the literature. Our radiographic measurements of 59 atypical and 218 ordinary fractures now exclude a difference larger than $10 \%$ in mean femoral cortical thickness (sum of lateral and medial) with $95 \%$ confidence.

\begin{abstract}
Purpose

An increased general cortical thickness in patients with fatigue fracture of the femoral shaft (atypical fractures) is commonly suggested. However, there is scarce data to support this.
\end{abstract}

\section{Methods}

In a published nationwide Swedish study, we identified by radiographic review 59 women with an atypical fracture during 2008. The femoral cortical thickness index (thickness / femoral diameter) of these women was now compared with the 218 ordinary fractures that occurred in the same region of the femur in a case control design. The cortical thickness index $5 \mathrm{~cm}$ below the lesser trochanter was the primary variable.

\section{Results}

Patients with atypical fractures were younger. Without correction for age, they had a thicker cortex (i.e. higher index). However, the difference in cortical thickness disappeared after age correction. The 95\% CI excludes a group mean difference exceeding $10 \%$ of total mean thickness. Similarly, there was no significant difference in cortical thickness between patients with or without bisphosphonate treatment, or between the ipsi- and contralateral femurs in patients with an atypical fracture.

\section{Conclusion}

The concept of a generally increased cortical thickness in patients with atypical fractures needs further assesment.

Keywords: Fractures, stress / bisphosphonates / osteoporosis / femoral fracture 


\section{Introduction}

Fatigue fractures of the femoral shaft, so called atypical fractures, are strongly associated with bisphosphonates[1], yet they are uncommon..A search for other contributing risk factors is, therefore, warranted. As these patients are generally younger[2] than others with subtrochanteric or femoral shaft fractures, and because the fracture is related to material fatigue, a high physical activity could be an important predisposing factor. Unknown genetic factors might also play an important role. It has been suggested that patients with atypical fractures have a thicker cortex than others[1,3,4]. A thick cortex is here regarded as a general phenomenon, and should not be mixed up with focal thickening at the fracture. Focal thickening appears to be a periosteal or endosteal callus reaction, developing around the undisplaced fatigue crack before it becomes a complete fracture.

A thicker cortex should be expected to reduce the fracture risk[5]. If it is correct that patients with atypical fractures have a thicker cortex than others, a thick cortex could signal some kind of material deficit due to genetic or other predisposition. It would consequently be an important marker for an increased risk of atypical fracture. Another possibility would be that a thicker cortex is just an effect of bisphosphonate use. In either case, if a thick cortex is associated with an increased risk of atypical fracture, it could be a relative counter-indication for starting or continuing bisphosphonate use.

The cortical thickness of the femur decreases with age[5,6]. It is therefore necessary to have a sufficiently large patient material to allow correction for this confounding factor. Our previously published material of 277 femoral shaft fractures, from a nation-wide population-based study[7], seemed to enable this. We now tested the hypothesis that patients with atypical fractures have a thicker cortex than patients with other types of subtrochanteric or femoral shaft fractures, by re-evaluating the radiographs of this previous study. 


\section{Material and Methods}

Study population

We measured the cortical thickness on radiographs, which were obtained for a previously published nationwide population-based study of atypical fractures. In that study, we investigated the radiographs of all 1234 women over 55 years of age in Sweden, who were registered as having sustained a femoral shaft fracture in the year 2008. We also obtained data from the National Swedish patient Register on the patients' drug use and comorbidities. Patients with high energy trauma were excluded. Of the 1234 radiographically reviewed cases, 277 met the criteria for inclusion, i.e. shaft fractures without major trochanteric involvement, implants or other skeletal pathology. 59 of these fractures were classified as atypical fractures in the previous study[7], where they were defined as fractures with a rather transverse orientation at the lateral cortex (fracture angle close to 90 degrees). 48 of them had focal thickening of the lateral cortex at the fracture site, and a few had a separate intermediate fragment. General cortical thickening was not used as a criterion to discern whether the fracture was atypical or not..

\section{$\underline{\text { Measurements }}$}

In the present study, we measured the cortical thickness on the radiographs of these 59 atypical fractures and the remaining 218 patients with other types of femoral subtrochanteric or shaft fractures. All measurements were undertaken by VK, blinded for any patient information, including medical background, drug history and former classification. Measurements were conducted on plain antero-posterior radiographs of the pelvis, hip or femur. In a few cases, because of outward rotation, the lateral radiographs were used because they gave a better frontal projection of the femur.

Measuring was carried out with the Sectra Workstation IDS7, Version 14.1.0.503, using the digital toolbox of this PACS software. We measured cortical thickness 5 and $10 \mathrm{~cm}$ below to the most prominent tip of the lesser trochanter. Cortical thickness was obtained by subtracting the medullary diameter from the shaft diameter .

First, two lines were drawn perpendicular to the femoral shaft axis 5 and $10 \mathrm{~cm}$ below the most prominent tip of the lesser trochanter. Along those lines we measured the width of the femoral shaft and the medullary cavity (Online Resource 1), the difference between these two being the cortical thickness. The ratio between cortical thickness and outer shaft diameter forms the cortical thickness index[8]. This unit-less index accounts for differences in radiographic magnification[1] and varying femoral size. A high index reflects greater cortical thickness and vice versa. 
Apart from the periosteal, beak-like callus formation near an atypical fracture, sometimes also an endosteal callus can be seen, typically extending several centimeters from the fracture. Measurements were made only on those levels ( 5 or $10 \mathrm{~cm}$ below the lesser trochanter) where such callus was absent.

When measurement on the fractured femur was not possible, values were substituted with measurements from the contralateral femur, when available. Of the total 277 patients, there were 140 patients (4 atypical) in whom cortical thickness could not be measured on the fractured femur at $5 \mathrm{~cm}$, mainly due to fracture characteristics, such as comminution. Of these 140 patients with missing ipsilateral data, 70 (no atypical) had contralateral values available (Table 1).

After these substitutions, 53 fractures remained (1 atypical) which could not be used in the analysis for any level. These were mainly cases with long spiral or communited fractures engaging both measuring levels, or cases with inappropriate projections or poor image quality. The number of appropriate measurements obtained at the different levels was 207 at $5 \mathrm{~cm}$ and 188 at $10 \mathrm{~cm}$.

\section{$\underline{\text { Statistical analysis }}$}

We used ANCOVA, with cortical thickness as dependent variable, atypical fracture or bisphosphonate use as independent variable and age as covariate. The measuring site with the highest number of appropriate measurements $(5 \mathrm{~cm})$ was used as the primary variable. The statistics program was IBM SPSS Statistics 19. 


\section{Results}

Cortical thickness index of the fractured femur $5 \mathrm{~cm}$ below the lesser trochanter, decreased with age $\left(\mathrm{r}^{2} 0.17\right.$; p 0.000). Patients with atypical fractures were younger (75 SD 10 years, versus 84 SD 9). Without correction for age, patients with atypical fractures appeared to have a higher cortical thickness index $(0.41$ SD 0.09, versus 0.37 SD 0.08; p 0.003). However, after age correction the difference in cortical thickness index became statistically insignificant $(95 \% \mathrm{CI}-0.01$ to +0.04 ; Table 1$)$. Values for $10 \mathrm{~cm}$ were similar (95\% CI -0.002 to +0.07). Patients using bisphosphonates had a higher cortical thickness index at $5 \mathrm{~cm}$ before (p 0.000), but not after age correction $(95 \%$ CI -0.02 to +0.03$)$.

In order to exclude that patients that were unfit for ipsilateral measurement had generally thinner cortices, we analyzed their contralateral values and compared them with data from patients $(n=82)$ who had both ipsi- and contralateral values available. With age correction there was no significant difference (95\% CI 0.03 to +0.02 ), suggesting that substitution with contralatera measurements did not cause bias.

For the atypical fractures, a side comparison at $5 \mathrm{~cm}$ (by paired t-test) was possible in 29 patients. There was no significant difference $(95 \% \mathrm{CI}-0.006$ to +0.04$)$.

The mean cortical thickness at $5 \mathrm{~cm}$ was $13 \mathrm{~mm}$, corresponding to a cortical thickness index of 0.38 . The confidence limit of 0.04 (more exactly 0.039) for the difference in index between atypical and ordinary fractures (see above) therefore corresponds to approximately $0.039 * 13 / 0.38=1.3 \mathrm{~mm}$, i.e. a $10 \%$ difference between the groups can be excluded. 


\section{Discussion}

Our results show no association between atypical fractures and general cortical thickness of the femoral shaft, although an analysis without age-correction erroneously gives such an impression. The 95\% confidence interval excludes that the medial and lateral cortex together are more than $1.3 \mathrm{~mm}(10 \%)$ thicker than controls at $5 \mathrm{~cm}$ below the lesser trochanter. This is a small difference, considering the variation in cortical thickness in the population (Fig. 1). At $10 \mathrm{~cm}$ the confidence interval is wider, but still excludes a substantial difference between the groups.

The term cortical thickening sometimes refers to focal cortical thickening, caused by the callus reaction at the site of a fatigue fracture, and sometimes to a general cortical thickness of the diaphyseal femoral shaft, seen as reflecting a premorbid condition. Unfortunately, the distinction between these two is often unclear in the literature. This paper only refers to the latter definition, i. e. general cortical thickness.

There are two hypotheses often mentioned regarding cortical thickness in the context of atypical fractures. First, that it is caused by bisphosphonates. This hypothesis has no support in our data, and has been refuted previously[6,9]. Secondly, that increased cortical thickness is a risk factor for atypical fracture[3,4]. This is an interesting thought, because if an independent risk factor could be identified, it could alter indications for bisphosphonate use. Moreover, it could have a bearing on the pathophysiology of atypical fractures. However, our data do not support this hypothesis, since a substantial difference was excluded.

Several case reports[10,11] about atypical fractures have mentioned that the cortices seem generally thick, nourishing the concern that an increased cortical thickness might be a risk factor. Atypical fractures have been defined as "simple with thick cortices" according to Lenart et al.[1], who found that patients on bisphosphonates with atypical fractures were younger and seemed to have thicker cortices. However, the authors did not connect cortical thickness with age, and it is unclear if they refer to focal or general thickening.

Although most authors only report their visual impression, Giusti et al. [6] actually measured cortical thickness. At $5 \mathrm{~cm}$ below the lesser trochanter, they found a significantly higher cortical thickness index in 10 atypical fractures (mean value of 0.49) compared to 53 controls (0.39). Their atypical fractures had thicker cortices than ours. This difference is hard to explain, but a thick cortex might have been part of their definition of atypical fracture ("fracture in an area of thickened cortices"), or they inadvertently included an endosteal callus in the measurement. Sometimes an endosteal callus reaction can extend several $\mathrm{cm}$ from an atypical fracture. In our study, this was avoided by measuring at another level when necessary. 
A prospective multicenter cohort study[5] showed that women with thicker cortices were at a lower risk of suffering from any kind of fracture of the femur, including subtrochanteric or shaft fractures. It makes sense that thicker cortexes are less likely to fracture.

A comprehensive review on atypical femoral fractures by the American Society for Bone and Mineral Research[3] summarized the features of those fractures in a chart with 5 major and 7 minor criteria. Increased general cortical thickening is one of those minor criteria. Our results suggest that this minor criterion should be reassessed.

The strength of the present study is mainly the fact that it is based on the entire Swedish population, which excludes selection bias, and that the material is sufficiently large to produce narrow confidence intervals for the difference between atypical and ordinary fractures. Another advantage is that we compared cases which all had subtrochanteric femoral fractures. A comparison of atypical fracture cases with controls without fractures, or with hip fractures, could have been prone to selection bias A further strength is the bilateral measurements at two different levels of the femur with consistent findings. The weaknesses include that the radiographs of $19 \%$ of the patients did not permit measurement on any level of the fractured side. Almost all these immeasurable cases belonged to the non-atypical group, and many of them had long, spiral fractures. However, the cortical thickness of the contralateral side of these cases was not different from the measurable ones, suggesting that the immeasurable cases did not cause bias due to a thinner cortex. A further weakness is that only women were studied, which does not take gender differences in cortical thickness into account. Finally, we did not differentiate between the medial and lateral cortices, as we regarded the sum of their thickness as the best measure of cortex thickness in general.

The absence of a substantial difference in cortical thickness between atypical and ordinary fractures suggests that we have to reconsider the idea that, an increased general cortical thickness indicates the presence of unknown factors, increasing the susceptibility for atypical fractures. 
Table

Table 1 Differences in cortical thickness index between atypical fractures and controls, after age correction

\begin{tabular}{|c|c|c|c|c|c|c|c|c|c|c|c|}
\hline & \multirow{2}{*}{$\begin{array}{l}\text { Total } \\
\mathrm{n}\end{array}$} & \multicolumn{3}{|c|}{ Atypical Fractures } & \multicolumn{3}{|c|}{ Control Fractures } & \multicolumn{4}{|c|}{ Difference } \\
\hline & & $\mathrm{n}$ & Mean & SE & $\mathrm{n}$ & Mean & SE & Mean & Sig. & CI low & CI high \\
\hline \multicolumn{12}{|l|}{$5 \mathrm{~cm}$} \\
\hline combined $^{1}$ & 207 & 55 & .39 & .011 & 152 & .37 & .011 & .014 & .291 & -.012 & .039 \\
\hline ipsilateral & 137 & 55 & .39 & .011 & 82 & .38 & .009 & .010 & .520 & -.020 & .040 \\
\hline \multicolumn{12}{|l|}{$10 \mathrm{~cm}$} \\
\hline combined $^{1}$ & 188 & 55 & .46 & .014 & 133 & .43 & .009 & .032 & .067 & -.002 & .066 \\
\hline ipsilateral & 148 & 52 & .46 & .014 & 96 & .44 & .010 & .021 & .233 & -.014 & .055 \\
\hline
\end{tabular}

${ }^{1}$ i.e. lacking ipsilateral values substituted with contralateral measurements. 
Figure

Fig. 1 Correlation of cortical thickness ratio with age in atypical and control fractures:

The scatter-plot shows a decrease in cortical thickness index with age. Atypical fractures tend to occur at a younger age.

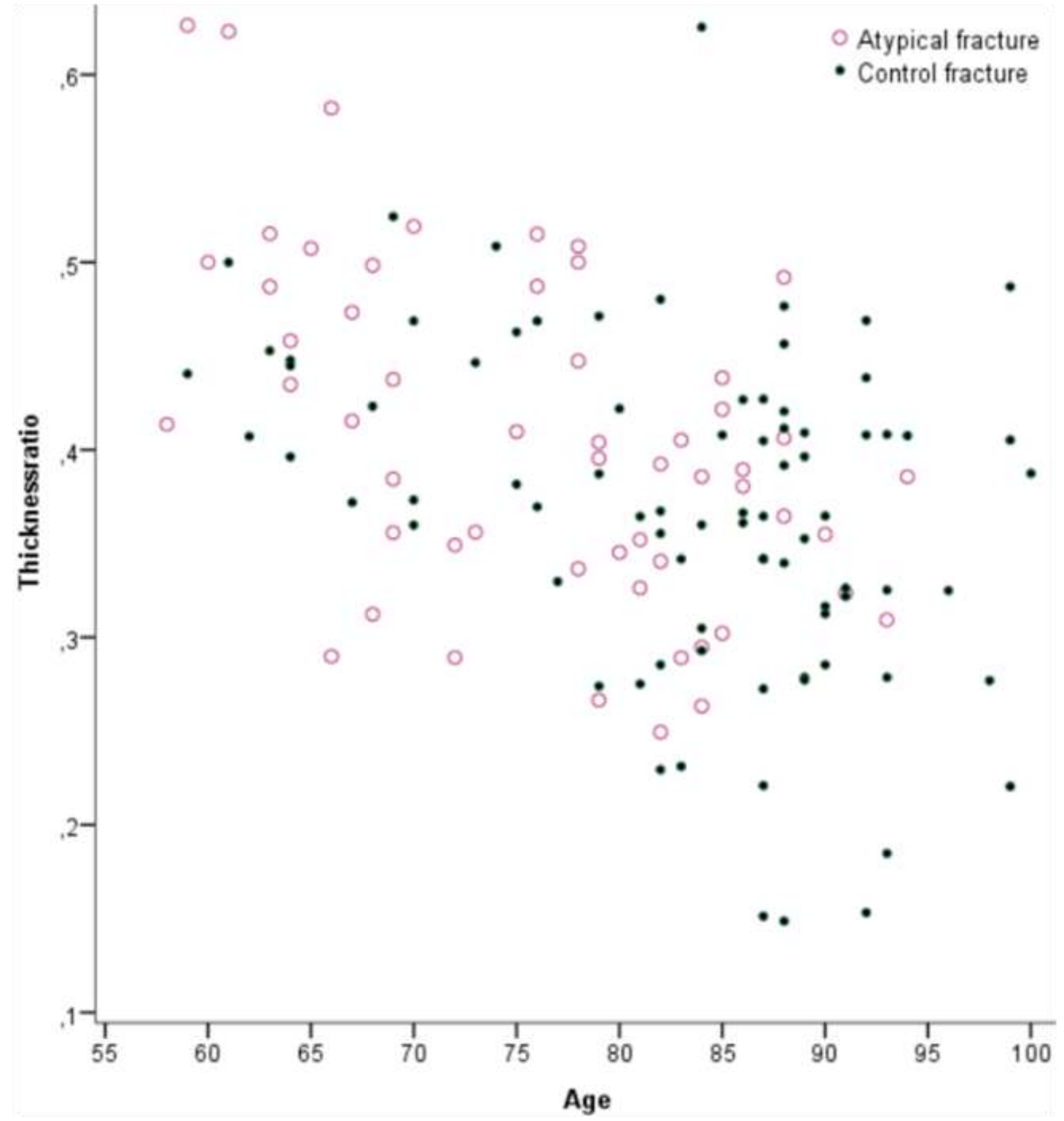




\section{Online Resource}

ESP 1 Measurement of cortical thickness at $5 \mathrm{~cm}$. The fracture is considered atypical. The endosteal contour is often somewhat blurred, and the measuring point was chosen at the border of the compact bone, as seen in higher magnification.

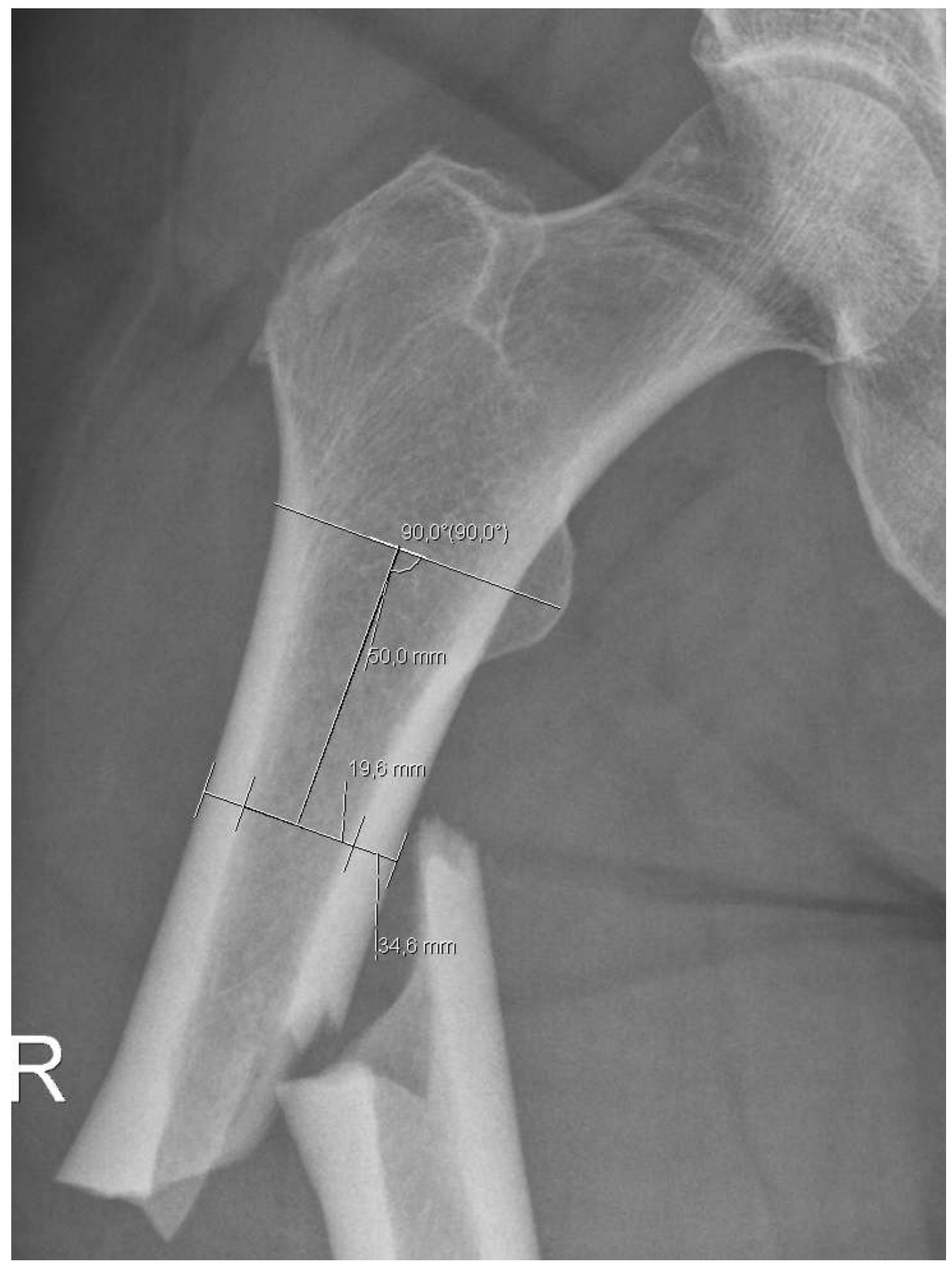




\section{References}

1. Lenart BA; Neviaser AS; Lyman S; Chang CC; Edobor-Osula F; Steele B et al. (2009) Association of low-energy femoral fractures with prolonged bisphosphonate use: a case control study. In: Osteoporos Int 20:1353-1362

2. Lo JC; Huang SY; Lee GA; Khandewal S; Provus J; Ettinger B et al. (2012) Clinical correlates of atypical femoral fracture. In: Bone 51:181-184

3. Shane E; Burr D; Ebeling PR; Abrahamsen B; Adler RA; Brown TD et al. (2010) Atypical subtrochanteric and diaphyseal femoral fractures: Report of a task force of the american society for bone and mineral Research. In: J Bone Miner Res 25:2267-2294

4. Rizzoli R; Akesson K; Bouxsein M; Kanis JA; Napoli N; Papapoulos SE et al. (2011) Subtrochanteric fractures after long-term treatment with bisphosphonates: a European Society on Clinical and Economic Aspects of Osteoporosis and Osteoarthritis, and International Osteoporosis Foundation Working Group Report. In: Osteoporos Int 22:373-390

5. Napoli N; Jin J; Peters K; Wustrack R; Burch S; Chau A et al. (2012) Are Women with Thicker Cortices in the Femoral Shaft at Higher Risk of Subtrochanteric Diaphyseal Fractures. In: Journal of Clinical Endocrinology \& Metabolism 97: E-pub ahead of print

6. Giusti A; Hamdy NAT; Dekkers OM.; Ramautar SR.; Dijkstra S; Papapoulos SE. (2011)Atypical fractures and bisphosphonate therapy: A cohort study of patients with femoral fracture with radiographic adjudication of fracture site and features. In: Bone 48:966-971

7. Schilcher J; Michaëlsson K; Aspenberg P (2011) Bisphosphonate Use and Atypical Fractures of the Femoral Shaft. In: N Engl J Med 364:1728-37

8. Sah AP; Thornhill TS; Leboff MS; Glowacki J (2007) Correlation of plain radiographic indices of the hip with quantitative bone mineral density. In: Osteoporos Int 18:1119-1126

9. Rizzoli R; Laroche M; Krieg MA; Frieling I; Thomas T; Delmas P; Felsenberg D (2010) Strontium ranelate and alendronate have differing effects on distal tibia bone microstructure in women with osteoporosis. In: Rheumatol Int 30:1341-1348

10. Kwek EBK; Goh SK; Koh JSB; Png MA; Howe TS (2008) An emerging pattern of subtrochanteric stress fractures: A long-term complication of alendronate therapy? In: Injury 39:224-231

11. Odvina CV; Levy S; Rao S; Zerwekh JE; Rao DS (2010) Unusual mid-shaft fractures during longterm bisphosphonate therapy. In: Clinical Endocrinology 72:161-168 


\title{
OSTEOPOROSIS INTERNATIONAL Authorship \& Disclosure Form
}

Atypical fractures do not have a thicker cortex

\author{
Article Title (first faw words) \\ First Author: Veronika Astrid Koeppen \\ E-mail: veronika.koeppen@googlemail.com
}

After submission of this agreement signed by all authors, changes of authorship or in the order of the authors listed will not be accepted by Springer.

\section{AUTHORSHIP}

I, the undersigned author(s), certify that:

- I have seen and approved the final version of the manuscript, and all subsequent versions.

- I have made substantial contributions to conception and design, or acquisition of data, or analysis and interpretation of data;

- I have drafted the article or revised it critically for important intellectual content.

I accept public responsibility for it, and believe it represents valid work. As an author of this article, I also certify that none of the material in the manuscript has been previously published, nor is it included in any other manuscript. I certify that this manuscript is not under consideration for publication elsewhere, nor has it been submitted or accepted in another publication in any form. The rights or interest in the manuscript have not been assigned to any third party.

Moreover, should the editor of Osteoporosis International request the data upon which the manuscript is based, I shall produce it. I also certify that I have read and complied with the copyright information, as found on the Osteoporosis International home page website.

\section{FINANCIAL DISCLOSURE/CONFLICT OF INTEREST}

I certify that any financial interests such as employment, stock ownership, honoraria, paid expert testimony, as well as any personal relationships, academic competition, and intellectual passion which may inappropriately influence my actions, have been disclosed on a separate attachment.

All funding sources supporting the work and all institutional or corporate affiliations of mine are acknowledged in a footnote.

I have had full access to all the data in the study (if applicable) and thereby accept full responsibility for the integrity of the data and the accuracy of the data analysis.

By checking the box next to my signature I assert that there are no conflicts of interest (both personal and institutional) regarding specific financial interests that are relevant to the work conducted or reported in this manuscript.

\section{PLEASE NOTE}

1. Every author must sign the Authorship \& Disclosure form.

2. It is possible to submit more than one form if the authors are in several locations.

3. All forms must be submitted at the same time.

4. Completed forms must be scanned and included as a pdf file during the online submission process as a supplemental file not for review.

Please email any queries to the appropriate Managing Editor:

European Office: Fina Liu - oi europe@iofbonehealth.org

USA Office: Adrianne Tewksbury - tewksburya@helenhayeshosp,org 


\section{OSTEOPOROSIS INTERNATIONAL Authorship \& Disclosure Form}

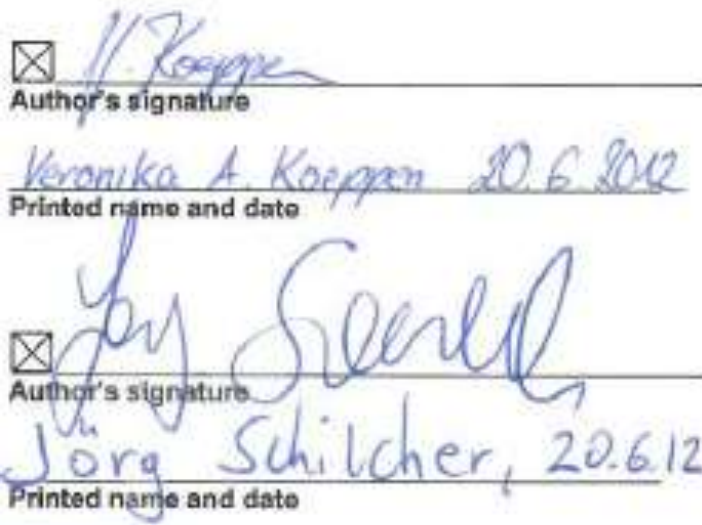

$\otimes$

Author's signature

Printed name and date

$\square$

Author's signature

Printed name and date

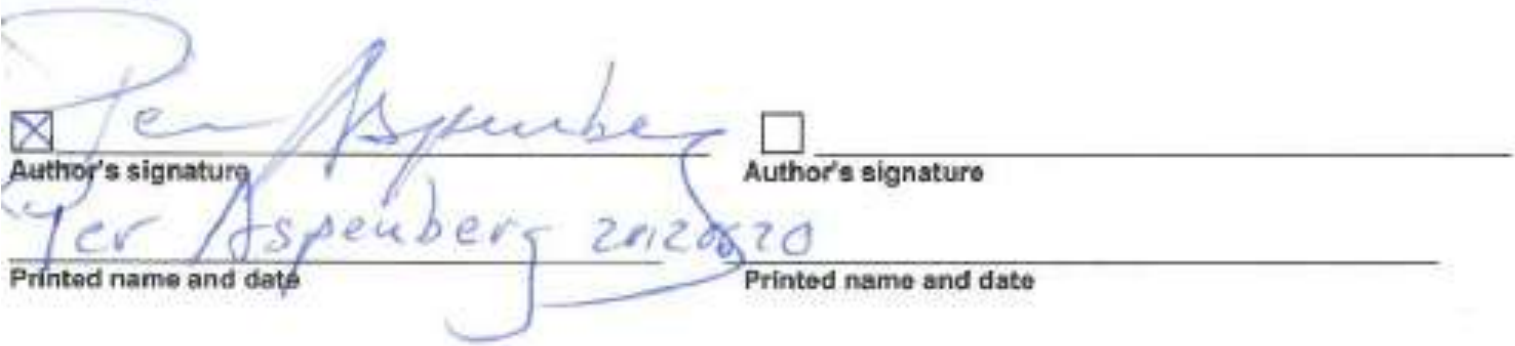

$\square$

Author's signature

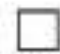

Author's signature

Printod name and date

Printed name and date

Author's signature

Author's signature

Printed name and date

Printed name and date

Author's signature

$\square_{\text {Author's signature }}$

Printed name and date

Printed name and dato

Page 2 of 2 - (signatures \& dates required on page 2) 